\title{
Diseño y construcción de un sistema electrónico para la repulsión de Copitarsia decolora (Lepidoptera: Noctuidae), plaga de flores de corte de exportación, mediante la simulación de llamadas de ecolocación de murciélagos
}

\author{
Desing and construction of an electronic system to Copitarsia \\ decolora repulsion (Lepidoptera: Noctuidae), kind of exportation \\ flowers plague, by means of simulation of bat echolocation calls
}

Fecha de recepción: 28 de marzo de 2008

Fecha de aceptación: 14 de julio de 2008
Hernán Paz Penagos', Marcela Rodríguez Ramírez ${ }^{2}$, Diana C. González ${ }^{3}$, Cesar M. Galarza4, Edison Torrado-León ${ }^{5}$.

\section{Resumen}

Una de las plagas interceptadas por el Servicio de Inspección de Salud de Animales y Plantas (APHIS) de Estados Unidos en las exportaciones de algunas especies de flores de corte de Colombia es Copitarsia spp. El presente estudio evaluó la respuesta evasiva de Copitarsia decolora (Lepidoptera: Noctuidae) mediante la simulación de llamadas de ecolocación de murciélagos como propuesta para el manejo en salas de poscosecha de flores, bajo condiciones controladas. Las formas de las señales de ecolocación de los murciélagos se tomaron de algunas muestras de quirópteros insectívoros y se simularon por medio del software Sound Ruler, y se determinaron los parámetros fundamentales que caracterizan las llamadas como su amplitud, frecuencia y forma, entre otros; a partir de esta caracterización, se diseñó y construyó un sistema electrónico capaz de reproducir las llamadas de ecolocación. Se probó el sistema, emitiendo la señal reproducida hacia una muestra de Copitarsia, y se observó una reacción evasiva frente a dicha señal.

\section{Introducción}

El valor de las exportaciones de flores representa el segundo renglón del sector agropecuario en Colombia, después del café, que, para el año 2003, fue de US\$806 millones. Una de las grandes limitaciones que tiene este

1 Magister en Teleinformática de la Universidad Distrital Francisco José de Caldas. Ingeniero Electricista de la Universidad Nacional de Colombia, Ingeniero Electrónico de la Universidad Distrital Francisco José de Caldas y Filósofo de la Universidad Santo Tomás de Aquino. Docente del Área de Comunicaciones de la Facultad de Ingeniería Electrónica de la Escuela Colombiana de Ingeniería Julio Garavito. E-mail: hpaz@ escuelaing.edu.co

2 Ingeniera Electrónica de la Escuela Colombiana de Ingeniería Julio Garavito. E-mail: marodrig@escuelaing.edu.co

3 Ingeniera Electrónica de la Escuela Colombiana de Ingeniería Julio Garavito.

4 Ingeniero Electrónico de la Escuela Colombiana de Ingeniería Julio Garavito.

5 Biólogo, Entomólogo. Profesor asociado de la Facultad de Agronomía de la Universidad Nacional de Colombia, Sede Bogotá; Línea de Investigación: Comportamiento de Artrópodos. E-mail: etorradol@unal.edu.co. 
mercado es el problema de las plagas ${ }^{6}$ que frecuentemente, de forma indeseable, acompañan al producto al destino de las exportaciones. El Servicio de Inspección de Salud de Animales y Plantas. (APHIS) de Estados Unidos, realiza revisiones sistemáticas a las flores exportadas a ese país para evitar la entrada de plagas cuarentenarias (Guerra y Forero, 2002); para aquellas plagas encontradas ${ }^{7}$ en los embarques de flores, especialmente lepidópteros del género Copitarsia ${ }^{8}$, se genera una fumigación, la devolución completa del cargamento o la incineración del mismo (Asocolfores, 2005).

En Colombia, las especies de Copitarsia son conocidas como "muques" o "cogolleros" y de acuerdo con el Instituto Colombiano Agropecuario -ICA- (1976), la especie registrada en Colombia es $C$. consueta (Walter, SD), siendo la sinonimia $C$. incommoda como la actualmente válida para esta especie (Angulo \& Olivares, 2003). Recientemente, estudios realizados por Torrado-León et al. (2005), demostraron que una de las especies presente en cultivos de flores de corte de la sabana de Bogotá es $C$. decolora.

En los cultivos de flores el manejo de estas plagas se realiza, generalmente, aplicando productos químicos o biológicos, con el fin de disminuir sus poblaciones y evitar que lleguen huevos o larvas a las salas de poscosecha. Sin embargo, estos métodos presentan un alto costo y generan daños en el medio ambiente. Este proyecto de investigación, que es patrocinado por la Fundación para la Promoción de la Investigación y la Tecnología del Banco de la República, y que se desarrolla interdisciplinariamente con la Facultad

6 El término genérico de $\square$ plaga $\square$, en la agricultura, designa a cualquier organismo que afecta a un cultivo, ya sea en forma directa o indirecta, causando pérdidas de importancia económica.

7 Entre las plagas interceptadas se encuentran pulgones, coléópteros, moscas, chinches, trips y polillas; éste último es el que presenta mayores interceptaciones y se reconoce dentro de este género a una polilla denominada Copitarsia.

$8 \mathrm{El}$ total de individuos de este género interceptados por APHIS entre enero de 2000 y marzo de 2002 de importaciones de flores procedentes de la sabana de Bogotá fue de 600 , para un total de $30.17 \%$ del total de las plagas reportadas (Guerra y Forero, 2002). de Agronomía de la Universidad Nacional de Colombia, sede Bogotá, hace una propuesta de control novedosa y altamente ecológica', que consiste en el diseño y construcción de un dispositivo electrónico de emisión de frecuencias de ultrasonido que simula la presencia del predador natural de la plaga, el murciélago, para impedir el ingreso de la Copitarsia decolora a los lugares de empaque de flores: las salas de poscosecha. En la primera y segunda fase del proyecto, ya concluidas, se realizó una revisión bibliográfica sobre la fisiología auditiva de la polilla y sus conductas frente a la presencia del predador; así mismo, se idèntificaron los tipos de señales, nivel de intensidad, duración y frecuencias que emiten las especies de murciélagos cuando cazan a sus presas ${ }^{10}$. Caracterizada la llamada de ecolocación, se diseñó, construyó y probó un sistema electrónico que simuló dicha señal. Toda la información relacionada a los anteriores aspectos se presenta de forma resumida en este artículo.

\section{Biología de C. decolora}

El-género Copitarsia decolora es de origen chileno, pero con el paso de los años ha ido extendiendo su presencia en todo el continente americano, mediante movimientos migratorios ocasionados por el fototactismo positivo que, al parecer, corresponde a un comportamiento de orientación con la reflexión de la luz que tienen las diferentes fases de la luna (Tucker, 1983; Angulo y Olivares, 2003). Se han registrado 21 especies para este género, distribuidas desde México hasta Chile.

Este Lepidóptero es un animal nocturno, cuenta con un sistema auditivo sofisticado que ha evolucionado

9 Un aspecto que no se ha estudiado es la repelencia de los adultos con sonidos simulados de sus enemigos naturales. Los machos y hembras de esta polilla presentan un par de tímpanos ubicados en el tórax, los cuales les han servido a todas las polillas de la familia Noctuidae y otros insectos, como un mecanismo de defensa o antidepredación, debido a que perciben los sonidos que emiten los depredadores para localizar sus presas (Norman et al., 1999).

10 Lalita Acharya and M. Brock Fenton: Bat attacks and moth defensive behaviour around street lights.(SD). 


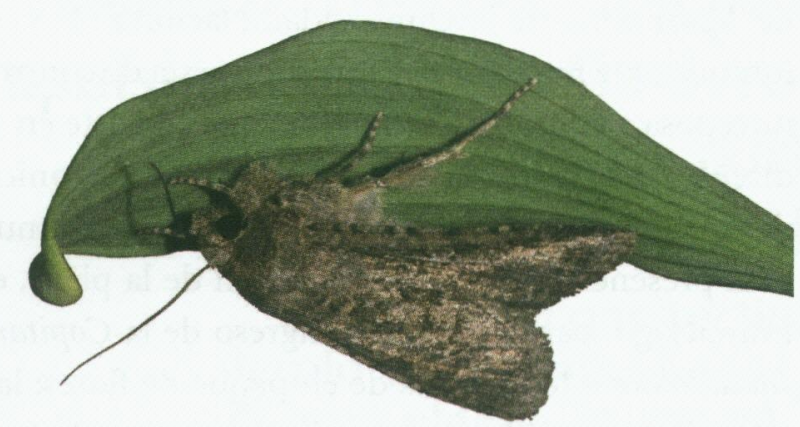

Figura 1. Adulto de Copitarsia decolora. Foto Edison Torrado-León $2006^{\circledR}$

con el paso de los años, de modo que le permite detectar la presencia del depredador (murciélago) y evadir su ataque realizando giros abruptos, vuelos en picada y otros movimientos instantáneos, como mecanismos de defensa (Norman et al., 1999).

Los oídos de las polillas son sensibles a sonidos en un amplio rango de frecuencias. Se considera que este rango está entre 3 y 150kHz. Así mismo, la sensibilidad auditiva de la polilla tiende a reflejar las frecuencias locales e intensidades de los sonidos de murciélagos (Waters, 1996; Jones et al., 2002; Fullard et al., 2003). Olivares y Angulo (1995) realizaron una descripción del tímpano de Copitarsia decolora (Copitarsia turbata), sin embargo, no describen las frecuencias de ultrasonidos en las cuales éstos son sensibles.

Las llamadas de ecolocación dependen de la especie, pueden ser señales moduladas en amplitud o frecuencia o señales pulsantes $(5 / 50 / 100$ pulsos/ seg.), de intensidad variable (75-110dB-SPL), corta duración (5-35mseg.) y alta frecuencia (15-70kHz); aunque cambian predeciblemente en dichas características a medida que se hace progresivo el ataque (Skals et al., 2003).

\section{El murciélago, depredador natural}

Los murciélagos son mamíferos pertenecientes a la orden de los Quirópteros y hay casi mil especies pertenecientes a quince familias alrededor del mundo, de las cuales tres son hematófagos, dos tercios insectívoros, un tercio frugívoros y nectívoros, y cerca de $1 \%$ carnívoros. La anatomía y morfología de los murciélagos están relacionadas con los hábitos alimenticios, lo que genera las distintas estructuras físicas que posee cada especie. Se ha considerado que estas formas se relacionan con la emisión de los llamados de ecolocalización.

Para la simulación del llamado de ecolocación del murciélago se estudiaron las especies Tadarida brasiliensis y Myotis chiloensis por su presencia en la Sabana de Bogotá; Sin embargo, se escogió la Tadarida brasiliensis (Molossidae) debido a que estudios realizados por Kunz et al. (1994), demuestran que su dieta depende en un 34\% de lepidópteros. La Tadarida brasiliensis morfológicamente es de estructura robusta de gran envergadura (aproximadamente $6.04 \mathrm{~cm}$ de longitud y $11.95 \mathrm{gm}$ de peso), con alas largas y angostas que le permiten un vuelo rápido y le impiden maniobrar en lugares cerrados como bosques. Sus velocidades medias ${ }^{11}$ se encuentran desde $6,0 \mathrm{~m} / \mathrm{s}$ (Hayward \&Davies, 1964) a 11,3 m/s (Svodova \& Choate, 1987). Por su parte, la especie Myotis chiloensis, se localiza en lugares cercanos a ríos, zonas áridas, bosques y sabanas. Este murciélago es pequeño, endémico, de aproximadamente de $9 \mathrm{~cm}$ de largo y $6 \mathrm{gm}$ de peso ${ }^{12}$; se caracteriza por tener un vuelo lento.

\section{Ecolocación}

El sistema de ecolocación es un mecanismo de percepción sensorial con el cual algunos animales se orientan, detectan obstáculos, se comunican entre sí y cazan, mediante la interrelación con el medio ambiente. Los llamados de ecolocación son sonidos generados en la laringe por la contracción de los músculos cricotiroideos y modificados con las formaciones bucales y nasales ${ }^{13}$; se basa en el

11 http://biomicro.sdstate.edu/pederses/Caribpics/Tbras.jpg.

12 http://www.scielo.cl/scielo.php?pid=S0716078X2001000300015 \&script=sci_arttext.

13 Seeing in the dark and tuning in with a bat detector por M. Brock Fenton, tomado de www.batcon.org/seedark.html\#one. 


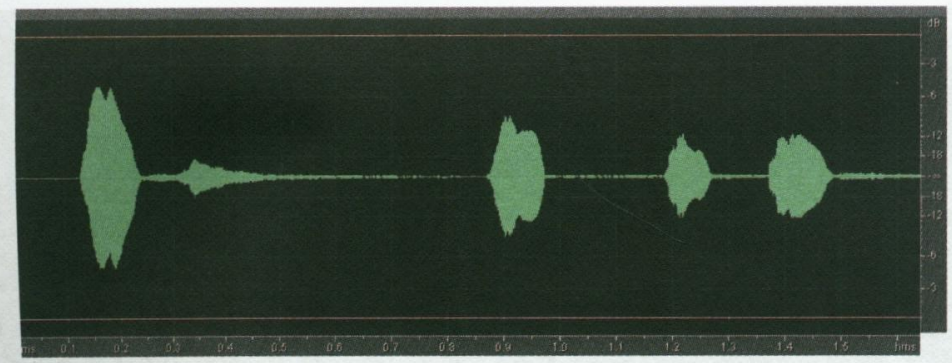

a

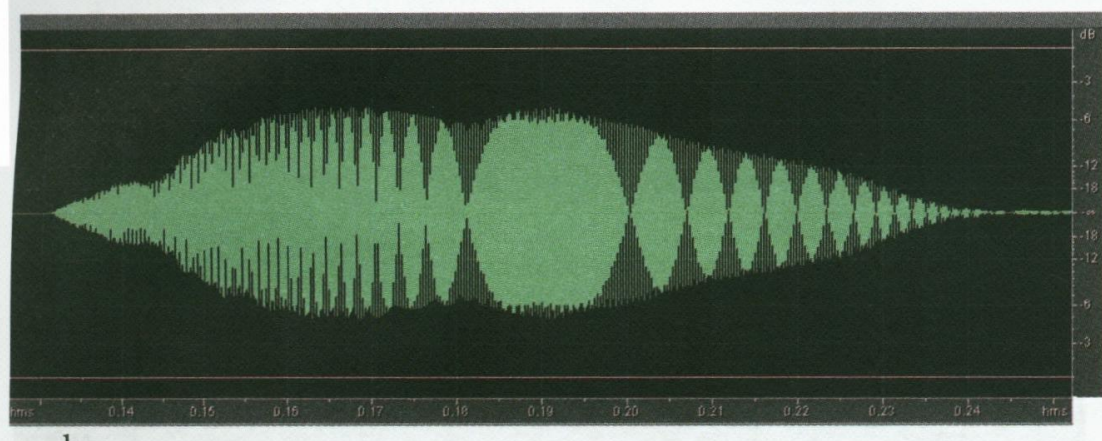

b

Figura 2. Llamada de ecolocación de especie Tadarida brasilensis (a) Envolvente de la señal modulada am, (b) Características de la señal portadora. Las señales de frecuencia contra tiempo fueron simuladas en Proteus 6 Professional.

Efecto Doppler ${ }^{14} \mathrm{y}$ son emitidas en frecuencias de ultrasonidos generalmente por encima del límite auditivo humano.

Cuando los llamados de ecolocación emitidos por el murciélago colisionan contra los objetos que se encuentran en su camino, se modifican y retornan en forma de eco con variaciones en sus parámetros: frecuencia, ángulo y amplitud; estas señales son captadas por las orejas, convertidas a señales eléctricas en los tímpanos y transmitidos al cerebro. Las diferencias relativas entre la información recibida y la señal emitida originalmente ${ }^{15}$, le permiten construir una representación exacta de los objetos de su entorno, lo que le da una ubicación espacial. Estos sonidos son usados especialmente por los murciélagos para localizar a su presa, identificar su forma,

14 El Efecto Doppler se basa en el siguiente fenómeno: "un observador situado en una posición fija, recibe las ondas sonoras desde una fuente en movimiento; el sonido producido será percibido en un tono más alto (esto es más agudo) a medida que la fuente se acerca al observador y en un tono más bajo (grave) a medida que se aleja", tomado de http://www.scielo.cl/scielo.php?pid=S0716078X2001000300015\&script=sci_arttext.

15 Tomado de www.batcalls.org. tamaño, velocidad y posición antes de atacar.

Las frecuencias de emisión del murciélago pueden variar desde $9 \mathrm{kHz}$ hasta $200 \mathrm{kHz}$ según la especie $^{16}$; la frecuencia de los llamados de ecolocación no es fija, aumen$\tan$ a medida que se acercan a sus presas. Por su parte el rango de escucha de las polillas es de $20 \mathrm{kHz}$ a $50 \mathrm{kHz}$. Dada esta diferencia, se puede afirmar que el sistema auditivo de las polillas no es sensible a los sonidos emitidos por todas las especies de murciélagos.

En las figuras 2a y $2 \mathrm{~b}$ se muestra una señal de cacería emitida por la especie Tadarida brasilensis, esta señal se caracteriza por estar modulada en amplitud, con una portadora de frecuencia variable que oscila entre $26 \mathrm{kHz}$ a $47 \mathrm{kHz}$.

La especie Myotis chiloensis genera señales de ecolocación de frecuencias altas: en el rango de $47 \mathrm{kHz}$ a $105 \mathrm{kHz}$ (véase figuras $3 \mathrm{a}$ y $3 \mathrm{~b}$ ).

Al comparar las características de cada una de las señales se puede confirmar que la señal más apropiada para continuar con la investigación es la emitida por la Tadarida brasiliensis, debido a que las señales de ecolocación se encuentra en el rango de frecuencias de $20 \mathrm{kHz}$ a $50 \mathrm{kHz}$, que corresponde al intervalo de escucha del lepidóptero Copitarsia decolora.

\section{Metodología}

Para el diseño y construcción del sistema electrónico que repele lepidópteros del género Copitarsia decolora se desarrollaron las siguientes etapas:

16 Seeing in the dark and tuning in with a bat detector por M. Brock Fenton, tomado de www.batcon.org/seedark.html\#one. 
1. Análisis de llamadas de ecolocación: fue necesario conocer la forma y los parámetros característicos de las señales de ecolocación de los murciélagos; por esta razón, se analizaron algunas muestras de las llamadas de murciélagos insectívoros usando el software Sound Ruler. La adquisición de las señales se efectuó con equipos especializados, como Anabat y Pettersson Elektronik, que permiten grabar señales de ultrasonido $(20 \mathrm{kHz}$ a $150 \mathrm{kHz}$ ), y trasladarlas a escala, a señales audibles para el ser humano $(20 \mathrm{~Hz}$ a $20 \mathrm{kHz})$. Estos equipos muestrean a $44.1 \mathrm{kHz}$ las señales análogas, las codifican en palabras de código de 16 bits y las almacenan en archivos digitales, con formato WAV; estas señales digitales se procesan en el software Proteus 6 Professional representando gráficas en varios dominios: amplitud-tiempo, frecuencia-tiempo, amplitudfrecuencia; sobre las cuales se puede identificar fácilmente los parámetros característicos de las señales de ecolocación.

En las figuras 4 a 6 se muestran las diferencias de los rangos de frecuencias y niveles de intensidad de las señales.

$\mathrm{Al}$ examinar las tres figuras anteriores se concluye que los murciélagos generan primero la señal de más alta frecuencia y la van disminuyendo en

a

b
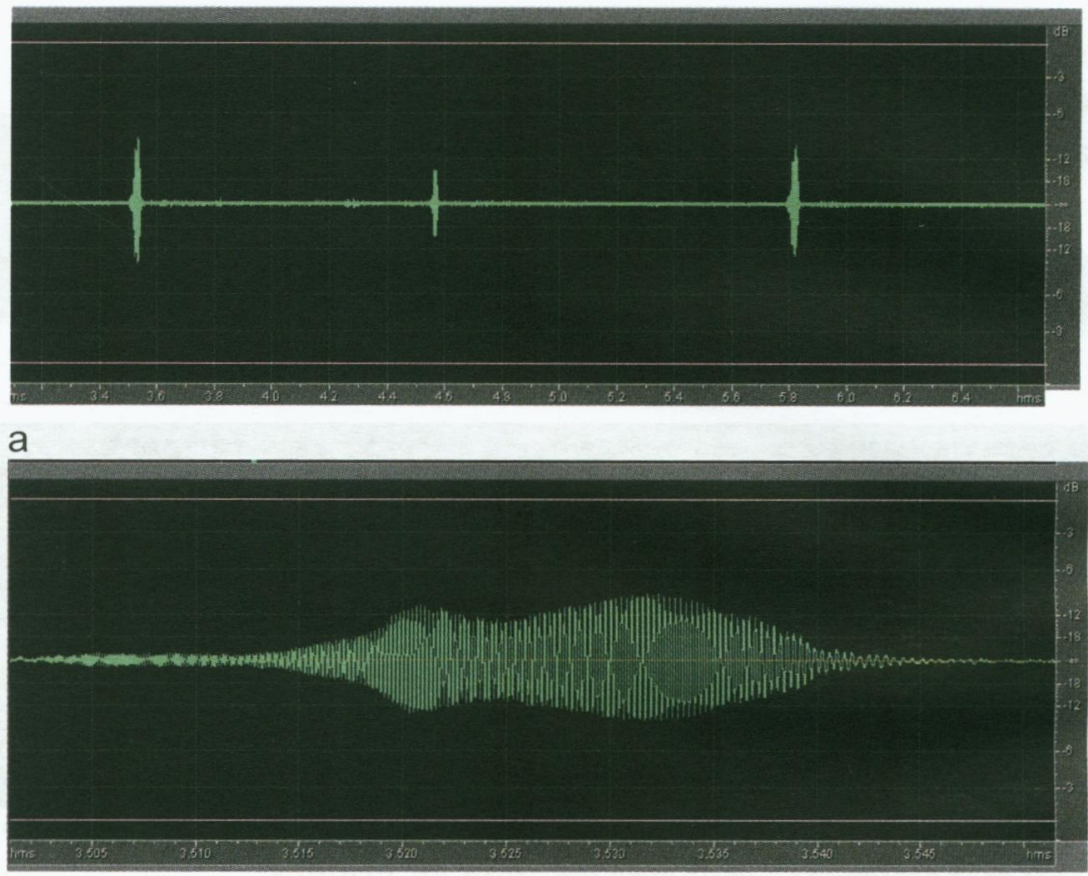

Figura 3. Señales de ecolocación de Myotis chiloensis (a) Envolvente de la señal modulada AM, (b) Características de la señal portadora. Las señales de frecuencia contra tiempo fueron simuladas en Proteus 6 Professional.

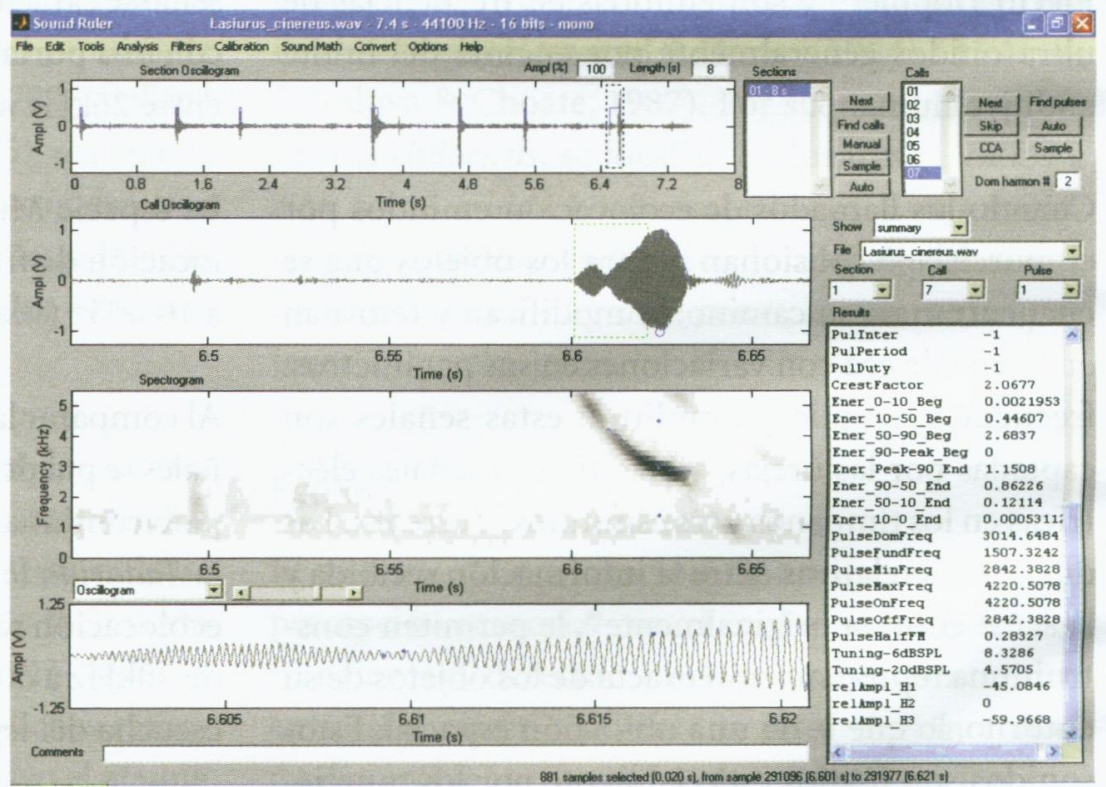

Figura 4. Señales emitidas por el murciélago de la especie Lasiurus cinereus, y procesadas en el software software Sound Ruler.

forma no lineal hasta alcanzar unas frecuencias mínimas (véase Tabla 1).

Otro parámetro importante de las señales de ecolocación, es la amplitud. En la figura 7, se 
representa una muestra de la señal de de la especie Eptesicus fuscus, que varía su amplitud desde un nivel de cero voltios hasta un valor máximo de 1 voltio, con un crecimiento no lineal; después decrece hasta alcanzar de nuevo el valor de cero. En dicha señal se reconoce un sistema oscilatorio con amplitud variable conforme transcurren el tiempo y las oscilaciones que no tienen la misma frecuencia. Por estas razones es posible concluir que se trata de una señal modulada en amplitud ${ }^{17}$.

2. Diseño de dispositivo: se basó en la simulación de las características principales de las llamadas de ecolocación de los murciélagos. Estas llamadas están compuestas por dos señales, una de baja frecuencia (modulante) y otra señal de alta frecuencia que realiza un barrido ultrasónico variable (portadora). Las etapas que conforman el dispositivo se muestran en el diagrama de bloques de la figura 8 .

Por medio del microcontrolador se generaron las señales portadora y moduladora. La señal portadora se conformó con un tren de pulsos cuadrados con frecuencia variable en un rango entre $26 \mathrm{k} \mathrm{y} 47 \mathrm{kHz}$ :

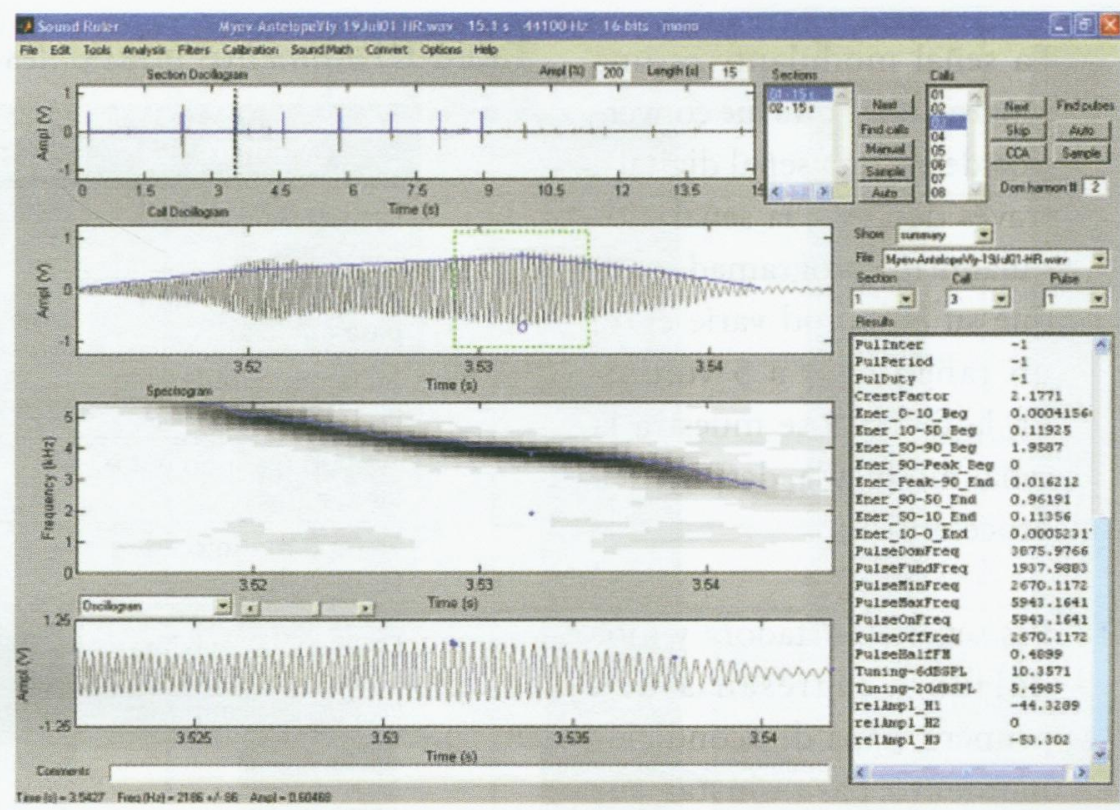

Figura 5. Señales emitidas por el murciélago de la especie Myotis evotis y procesadas en el software software Sound Ruler

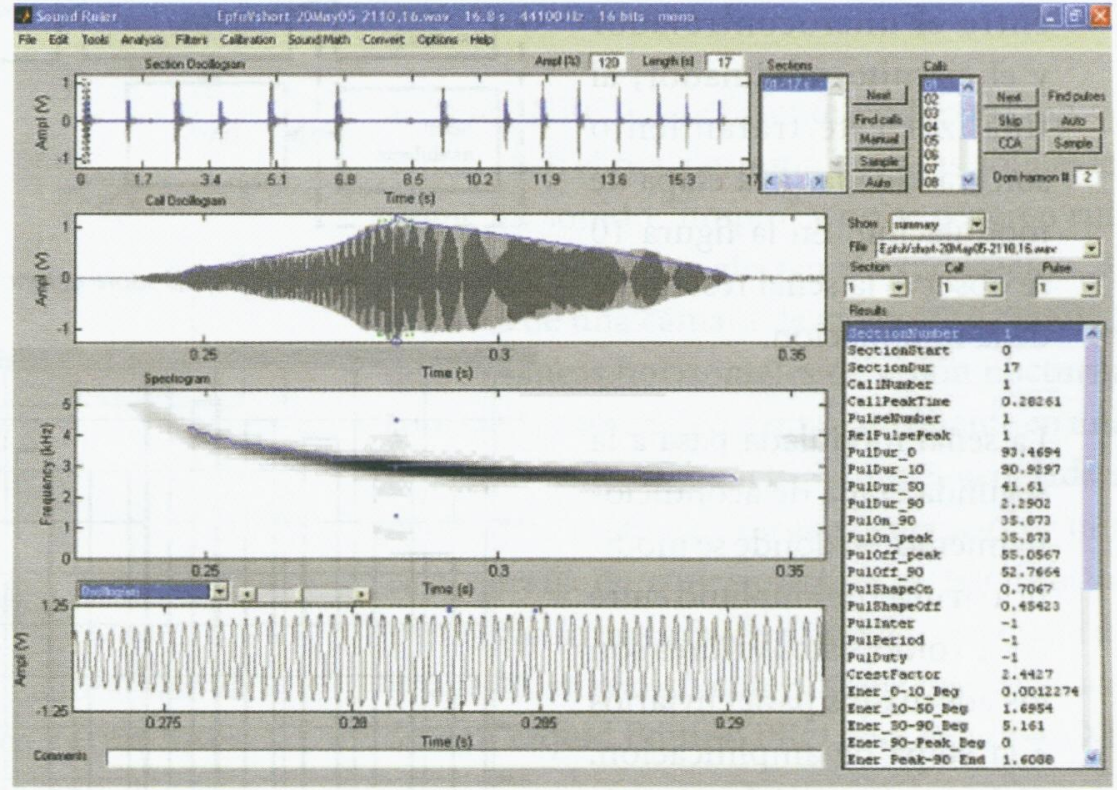

Figura 6. Señales emitidas por el murciélago de la especie Eptesicus fuscus y procesadas en el software Sound Ruler.

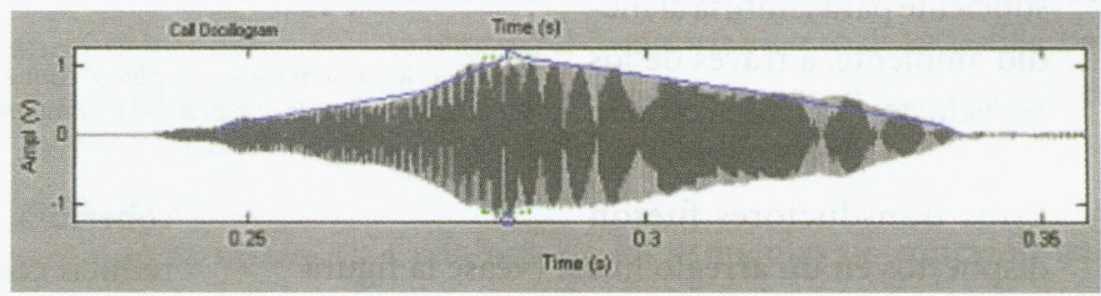

Figura 7. Representación (amplitud contra tiempo) de la señal emitida por la especie Eptesicus fuscus, cuando caza. Señal simulada en el software Sound Ruler.

17 La modulación en amplitud consiste en modificar la amplitud de una señal de alta frecuencias conforme cambia la señal modulante de baja frecuencia. 
La señal modulante es una señal análoga que fue convertida desde una señal digital a través de DAC; la señal modulante fue programada para que su amplitud varíe entre un rango de 4 a 5 voltios. En la figura 9 se muestra la señal modulada, después del procesamiento.

Las señales portadora y moduladora ingresan a una primera etapa de acondicionamiento para ajustar sus respectivas amplitudes, $\mathrm{y}$ de acoplamiento de impedancias entre el microcontrolador y el circuito modulador; al finalizar este tratamiento son trasladadas a la etapa de modulación. En la figura 10 se observa la señal resultante de la modulación.

La señal modulada pasa a la segunda etapa de acondicionamiento, en donde se modifica su nivel de amplitud entre 0 y 1 voltio; estos valores son los adecuados para enviarlos a la etapa de amplificación. El factor de amplificación de esta etapa es de 30 voltios, suficiente para emitirla al medio ambiente, a través de los transductores piezoeléctricos.

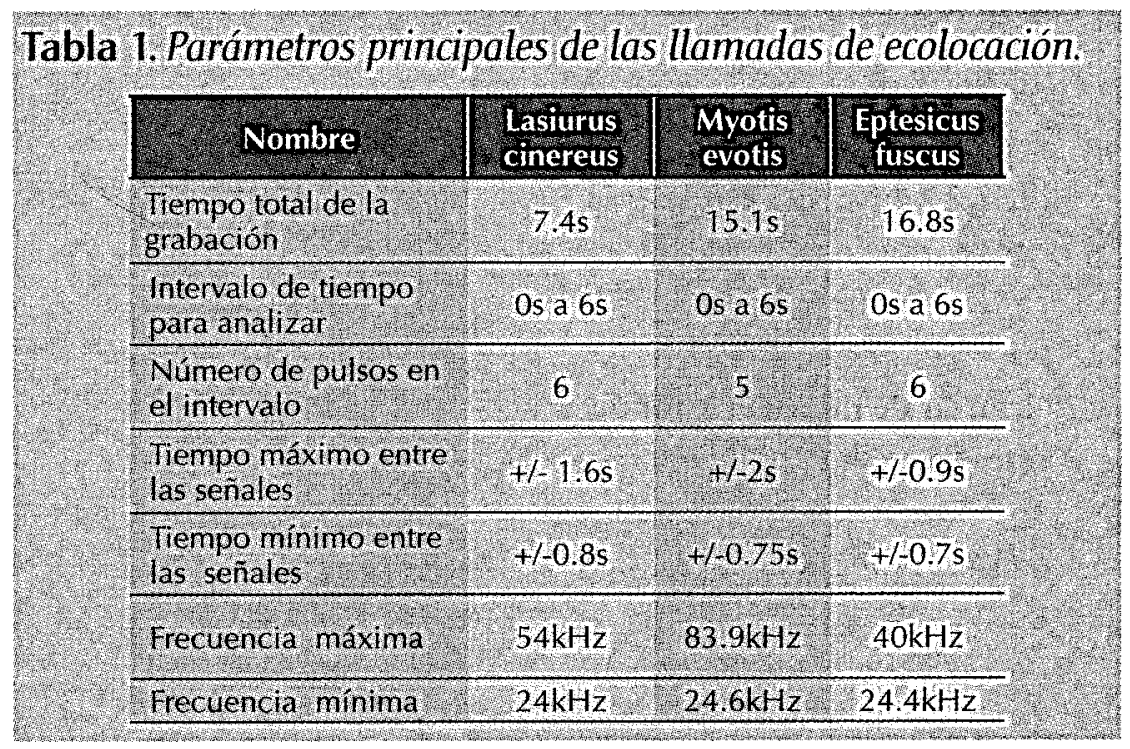

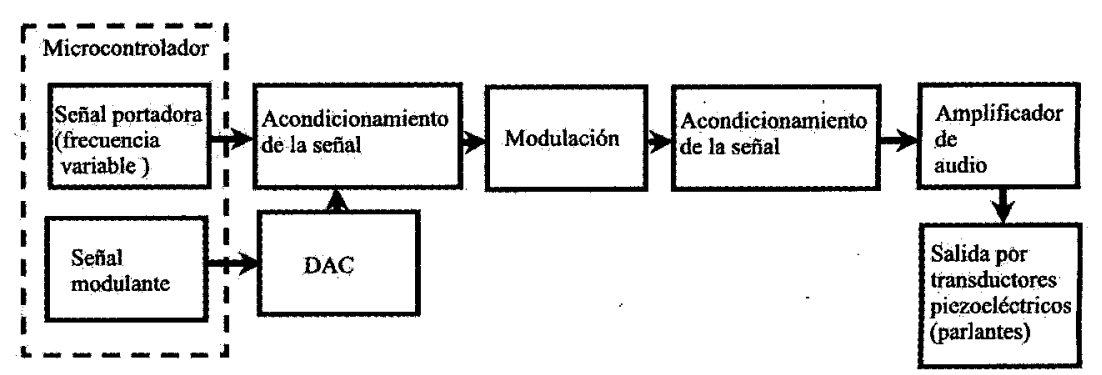

Figura 8. Diagrama de bloques sobre el funcionamiento del dispositivo.

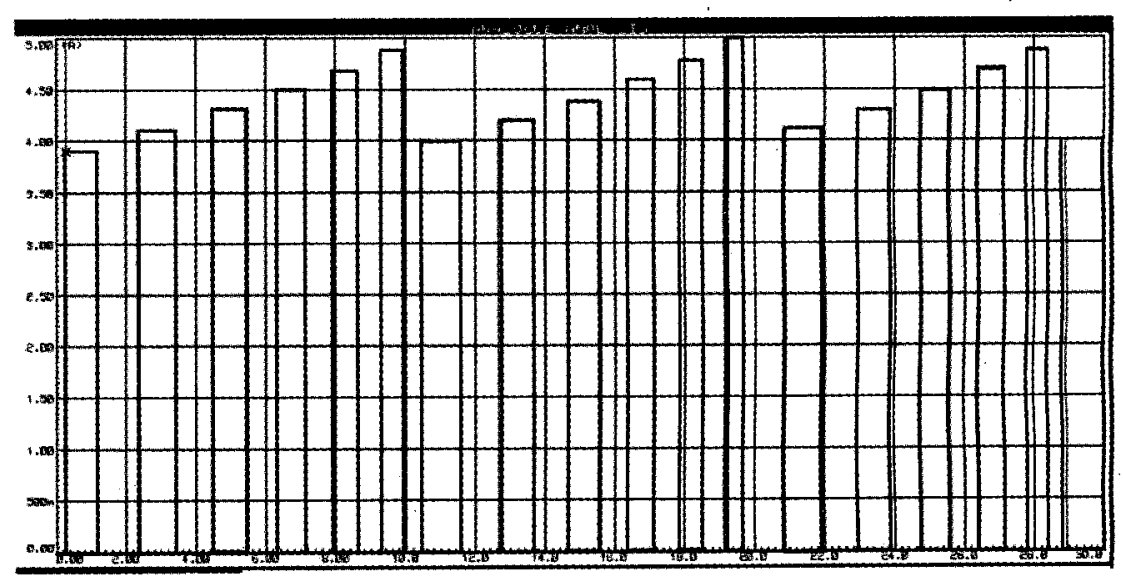

Figura 9. Representación (amplitud contra tiempo) de la señal modulada en amplitud. Simulación realizada con el software Proteus 6 Professional.

\section{Estos transductores fueron}

dispuestos en un arreglo lineal (véase la figura 11), en el cual los elementos son centrados a lo largo de una línea recta con igual distancia; esta red tiene como objeto maximizar el área de cobertura, optimizar el numero de parlantes, reducir costos, y usar adecuadamente el medio de propagación de la señal, para finalmente representar una fuente de emisión de llamadas de ecolocación simuladas. 


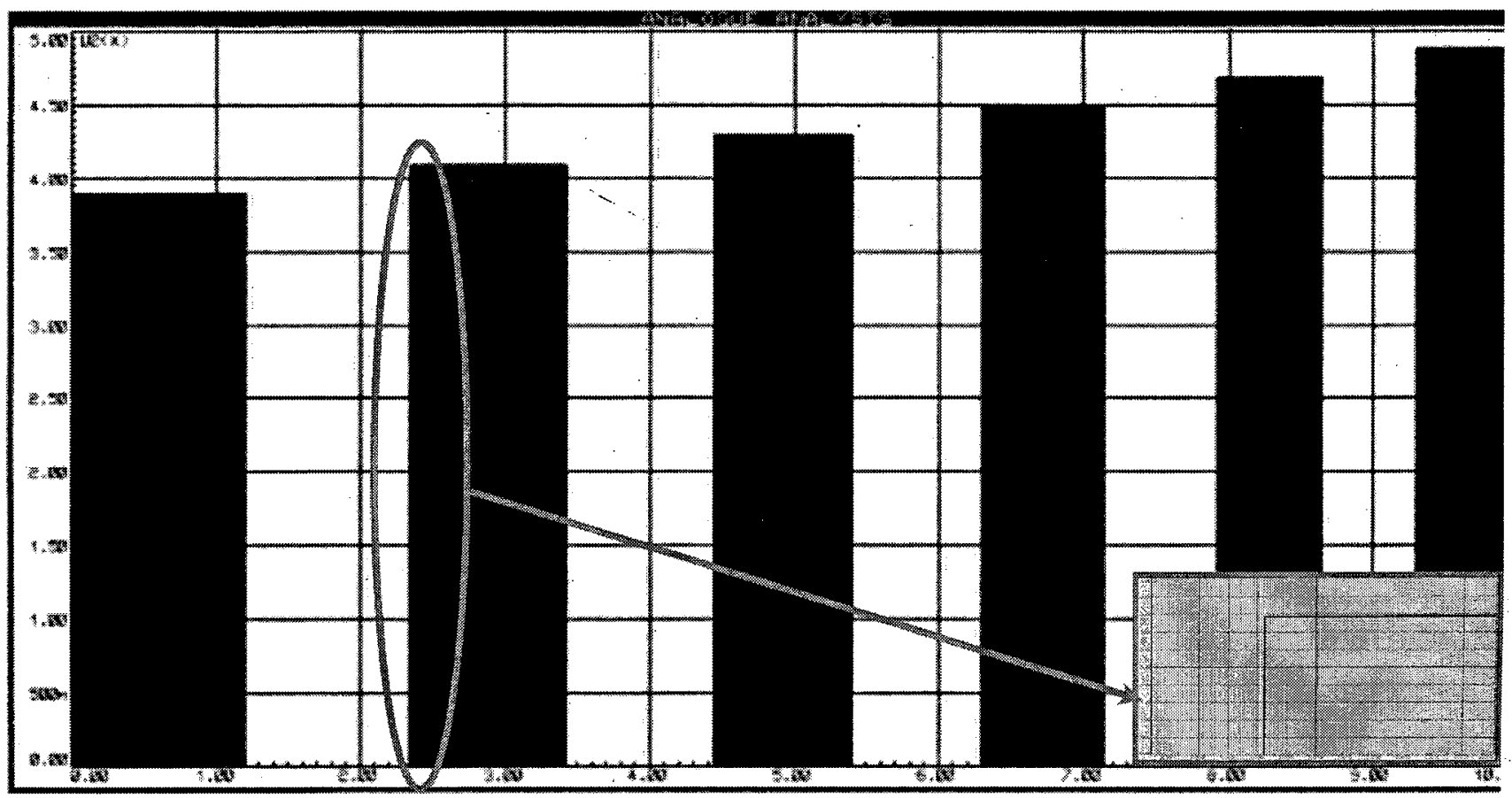

Figura 10. Señal registrada al concluir la modulación. Simulación (amplitud contra tiempo) realizada con Proteus 6 Professional.

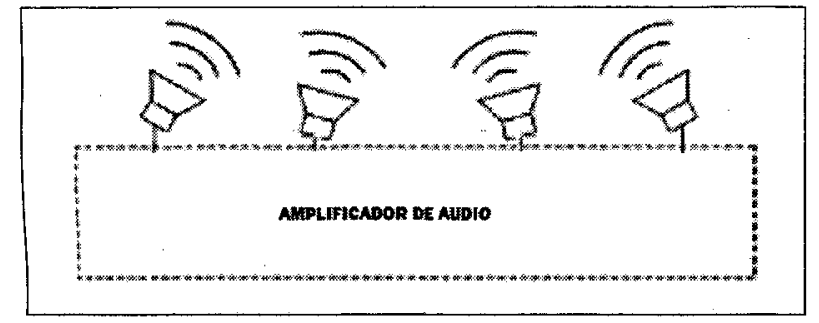

Figura 11. Red lineal de parlantes.

3. Pruebas:

Ubicación: se realizaron en el Laboratorio de Comportamiento de Artrópodos y otros Invertebrados de la Facultad de Agronomía, Universidad Nacional de Colombia, sede Bogotá.

Materiales: 1) Se evaluaron 3 especímenes del género Copitarsia decolora de no más de tres días de edad, 2) Cabina controlada de 100X50X60 $\mathrm{cm}$, con paredes de 1 " en tela negra no tejida en fibra de vidrio Black Theater ${ }^{\circledR}$; este es un sistema acústico y lumínico con propiedades de aislamiento térmico y acústico; el material con el que fue construido tiene una absorción acústica NRC - Coeficiente de Reducción de Ruido - 0.7; el cual permitió que a las polillas les llegue un sonido "limpio" y no se generen ruidos adicionales que afecten el experimento, 3) A través de una cámara de video $15 \mathrm{CC} 35 \mathrm{NV}-1$ de 600 Líneas horizontales de visión nocturna conectada a un medio digital consistente en una tarjeta de video Pinnacle System ${ }^{\circledR}$, acoplada a una tarjeta gráfica de última generación y una caja externa de conexiones, se registraron los eventos de la emisión de sonidos. La captura de imágenes se realizó con el software licenciado System Liquid Edition PRO para Windows XP.

Procedimiento: Se liberaron tres ejemplares de Copitarsia decolora con el dispositivo activado y se registró el comportamiento de las polilla; para el desarrollo de las pruebas se siguieron cuatro esquemas de conexión (véase la figura 12).

Cada uno de los esquemas de conexión tenía un protocolo de mediciones con un objetivo e hipótesis (véase Tabla 2). El sistema se evaluó en el transcurso del día y con un recinto oscuro, que simula horas de la noche. 
Tabla 2. Detalles del protocolo seguido con los cuatro esquemas de conexión.

\begin{tabular}{|c|c|c|c|}
\hline . & (201010100 & 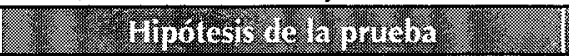 & 30 \\
\hline & $\begin{array}{l}\text { Realizar un barrido de la } \\
\text { frecuencia desde } 10 \mathrm{~Hz} \text { hasta } \\
100 \mathrm{kHz} .\end{array}$ & $\begin{array}{l}\text { Es posible que en el rango de } \\
\text { trecuencias entre } 10 \mathrm{lz} \text { a } 100 \mathrm{kH} \text {, el } \\
\text { Lepidóptero escuche al depredador. }\end{array}$ & $\begin{array}{l}\text { Generador de señales HM8030-s } \\
\text { HAMEG. } \\
\text { Fuente de alimentación } 33 \mathrm{~V} \text {, } \\
12 \mathrm{Vdc},-12 \mathrm{Vdc} \text {. } \\
\text { Amplificador de potencia, } \\
\text { transductor piezoeléctrico. }\end{array}$ \\
\hline & $\begin{array}{l}\text { Realizar un barrido de } \\
\text { frecuencia desde } 10 \mathrm{~Hz} \text { hasta } \\
100 \mathrm{kHz} \text { y orientar el transductor } \\
\text { con distintos ángulos hacia } \\
\text { la polilla, siguiendo un radio } \\
\text { circular con centro en el } \\
\text { espécimen. }\end{array}$ & $\begin{array}{l}\text { Es posible encontrar un angulo preciso } \\
\text { de ubicación de parlante en el que la } \\
\text { polilla perciba con mayor intensidad la } \\
\text { presencia del depredador }\end{array}$ & $\begin{array}{l}\text { Generador de señales HM8030-s } \\
\text { HAMEG. } \\
\text { Fuente de alimentación } 33 \mathrm{~V}, \\
12 \mathrm{Vdc},-12 \mathrm{Vdc} \text {. } \\
\text { Amplificador de potencia. } \\
\text { Transductor piezoeléctrico }\end{array}$ \\
\hline & $\begin{array}{l}\text { Someter al espécimen de } \\
\text { prueba a una señal modulada } \\
\text { en amplitud con varias señales } \\
\text { moduladoras senoidal, } \\
\text { cuadrada, triangular. }\end{array}$ & $\begin{array}{l}\text { E } \\
\text { Es posible que una señal modulada en } \\
\text { amplitud con una senoidal, trangular } \\
\text { o cuadrada simule correctamentela } \\
\text { senar del murciélago y se convierta en } \\
\text { una senal que los Lepidópteros evadan } \\
\text { por instinto de conservación. }\end{array}$ & $\begin{array}{l}\text { Generador de señales y } \\
\text { modulador Hung Chang (model } \\
\text { G 305). } \\
\text { Modulador MC1496. } \\
\text { Generador de señales HM8030-s } \\
\text { HAMEG. } \\
\text { Fuente de alimentación } 33 \mathrm{~V}, \\
12 \mathrm{Vdc},-12 \mathrm{Vdc} \text {. } \\
\text { Amplificador de potencia. } \\
\text { Transductor piezoeléctrico } \\
\end{array}$ \\
\hline 4 & $\begin{array}{l}\text { Estimular al espécimen con una } \\
\text { señal modulada en amplitud y } \\
\text { pulsante. }\end{array}$ & $\begin{array}{l}\text { Es posible que una señal } \text { grodulada. } \\
\text { y pulsante de forma seno, triangular y, } \\
\text { cuadrada se aproxime más a la señal } \\
\text { del murcielago real, y sea percibida } \\
\text { con mayor claridad por el Lepidóptero, } \\
\text { de tal manera que la evada con mayor } \\
\text { pronitud. }\end{array}$ & $\begin{array}{l}\text { Modulador MC1496, } \\
\text { Multiplexor } 4051 \text {, Generador } \\
\text { de señales HM8030-s HAMEG, } \\
\text { Fuente de alimentación } 33 \mathrm{~V}, \\
12 \mathrm{Vdc},-12 \mathrm{Vdc} \text {, Amplificador } \\
\text { de potencia, Transductor } \\
\text { piezoeléctrico. }\end{array}$ \\
\hline
\end{tabular}
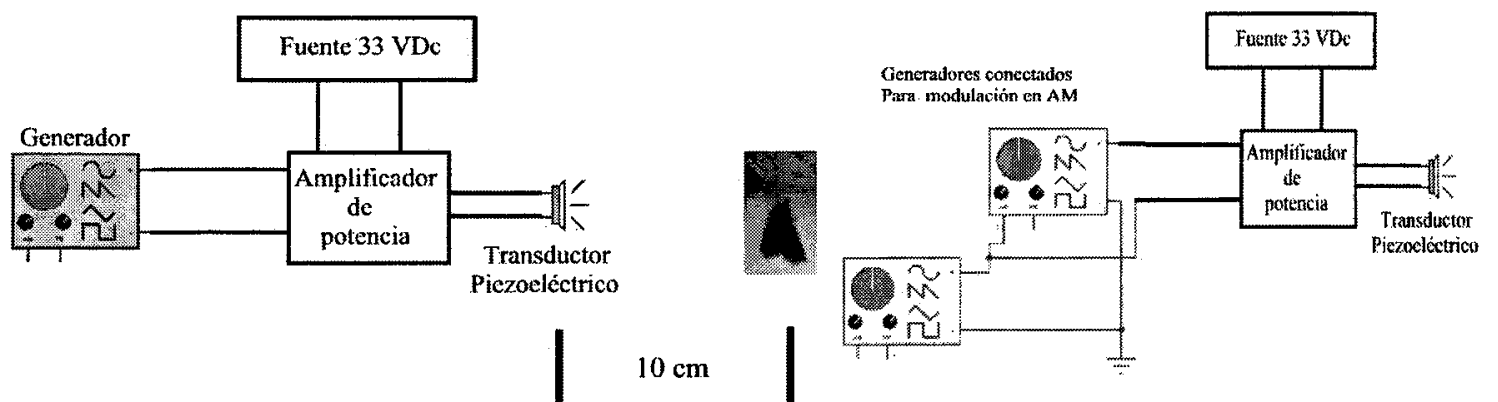

a

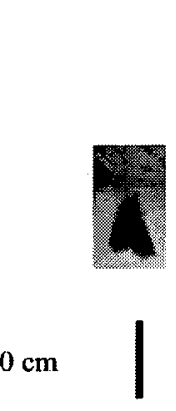

\section{$\mathrm{b}$}

Generador señal moduladora
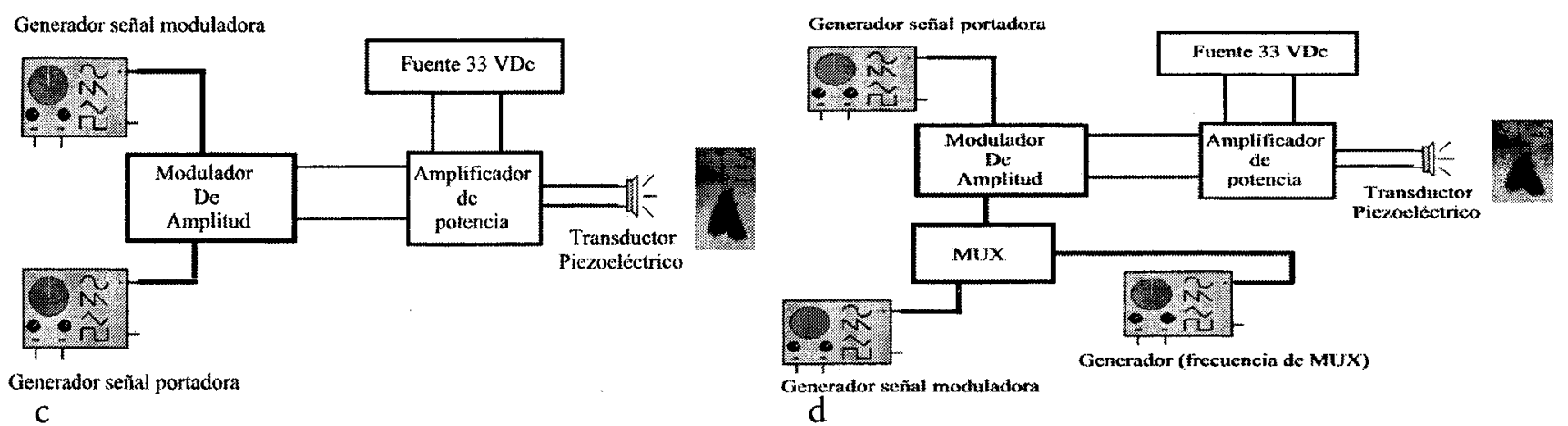

Figura 12. Esquemas de conexión del sistema electrónico que simula las llamadas de ecolocación del murciélago: a) Con un generador, b) Con dos generadores, c) Con dos generadores y un modulador externo, d) Con tres generadores y dos circuitos modulador y multiplexor externo. 
Tabla 3. Límites máximos de exposición según la frecuencia de operación.

\begin{tabular}{|c|c|c|c|}
\hline 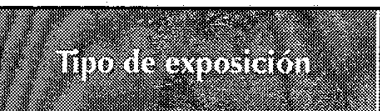 & 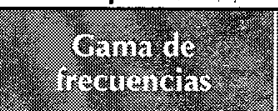 & 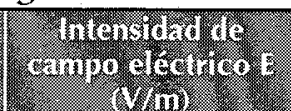 & $\begin{array}{l}\text { Trenstiod ge } \\
\text { campo nagneteo }\end{array}$ \\
\hline & $65 \mathrm{KL}$ & 610 & 24,4 \\
\hline
\end{tabular}

Fuente: Decreto 195 del 31 de enero de 2005 (tiene por objeto adoptar los límites de exposición de las personas a los campos electromagnéticos producidos por estaciones radioeléctricas en la gama de frecuencias de 9 $\mathrm{kHz}$ a $300 \mathrm{gHz}$ ). de acuerdo a las estadísticas existentes en otras aplicaciones con emisiones de ultrasonido en bandas de frecuencia similares (dispositivos electrónicos para repulsión de roedores, sensores mediante el principio de ultrasonido, etc.) es tan insignificante que no impide el desarrollo del dispositivo y la puesta en funcionamiento; Por otra parte, las emisiones de radiación de los transductores electrónicos (parlantes) no superan los límites de intensidad de señal máximos permitidos por la tabla 3.

\section{Conclusiones}

- El sistema auditivo de las polillas puede ser estimulado por medio de señales en el rango de ultrasonido.

- El rango estimado según las pruebas es desde $28 \mathrm{kHz}$ hasta $47 \mathrm{kHz}$ aproximadamente.

- Los movimientos halares vistos y registrados corresponden a los esperados; dentro de estos movimientos se destaca el bloqueo de los músculos halares y los vuelos erráticos que realizan al ser expuestos a la señal de prueba.

das las formas de energía radiante, distintas de las radiaciones ionizantes que se originen en lugares de trabajo, deberán someterse a procedimientos de control para evitar niveles de exposición nocivos para la salud o eficiencia de los trabajadores".

Sin embargo, se puede afirmar que el impacto de las ondas de ultrasonido ${ }^{18}$ sobre la salud de los trabajadores que empacan las flores en los cuartos de poscosecha es una posibilidad, pero que la incidencia,

18 El ser humano puede escuchar sonidos en el rango de $20 \mathrm{~Hz}$ a $20 \mathrm{KHz}$, mientras que los perros y los gatos tienen capacidad auditiva hasta 27 kilociclos por segundo.

CENTRO DE INVESTIGACIONES Y DESARROLLO CIENTÍFICO

\section{Bibliografía}

Angulo, A. O. \& T. S. Olivares. 2003. “Actualización taxonómica de las especies de Copitarsia Hampson 1906, (Lepidoptera: Noctuidae: Cucullinae)", en Gayana Zoología, 67 (1):33 - 38.

Asocolflores. 2005. "Procedimientos para la inspección fitosanitaria en Miami: Mejor estar informados para evitar fumigaciones innecesarias", en Noticias Frescas, 185: 15. 
Coughlin, R. F. \& F. F. Driscoll. 1999. Amplificadores operacionales y circuitos integrados lineales, cuarta edición. México, D.F: Prentice-Hall.

Fullard, J.H., K.E. Muma \& J.W. Dawson. 2003. "Quantifying an anti-bat flight response by eared moths" en Candian Journal of Zoology, 81: 395-399.

Guerra, J. J., \& D. Forero. 2002. “Principales interceptaciones de plagas en flores provenientes de la Sabana de Bogotá en el puerto de Miami, y su implicación fitosanitaria" en Revista Asocolflores, 62: $50-58$.

ICA. 1976. "Lista de insectos dañinos y otras plagas en Colombia" en Boletín Técnico Instituto Colombiano Agropecuario No. 43. 484 pp.

Jones, G., A. Barabas, W. Elliott \& S. Parsons. 2002. "Female Greater Wax Moths Reduce Sexual Display Behavior In Relation To The Potential Risk Of Predation By Echolocating Bats" en Behavioral Ecology, 13 (3): 375-380.

Kunz, J.O. Whitaker, J.R. y Wadanoli, M.D. 1994. Dietary Energetics Of The Insectivorous Mexican Free-Tailed Bat (Tadarida brasiliensis). 28 October 1994.

Millman \& Grabel. 1987.Microelectronics,segunda edición. McGraw Hill,.

Norman, A. P., G. Jones \& R. Arlettaz. 1999. "Noctuid Moths Show Neural And Behavioural Responses To Sounds Made By Some Bat-Marking Rings" en Animal Behaviour, 57: 829-835.

Proakis, J. 2001. Digital Communications, cuarta edición. Boston: McGraw-Hill.

Savant, Roden, Carpenter. 1992. Diseño electrónico, Segunda edición, Addison Wesley Iberoamérica.
Skals, N., D. Plepys,A. M. El-Sayed, C. L. Ofstedt \& A. Surlykke. 2003. "Quantitative Analysis Of The Effects Of Ultrasound From An Odor Sprayer On Moth Flight Behavior" en Journal of Chemical Ecology, 29 (1): 71-82.

Schuler, C. \& Chugani, M. 2005. Digital Signal Processing: A Hands-On Approach. Boston: McGraw-Hill.

Tomasi, W. 1996. Sistemas de comunicaciones electrónicas, tercera edición. México, D.F.: Prentice Hall.

Torrado-León, E., R. Lee, F. Serna, N. Quimbayo \& S.R. Muñoz. 2005. "Reconocimiento de Noctuidae (Lepidoptera) plaga en cultivo de flores". Memorias VIII Congreso Técnico Flores 2005, Bogotá, 26 de agosto, pp. 40-42.

Tucker, M. R. 1983. "Light-Trap Catches Of African Armyworm Moths, Spodoptera Exempta (Walker) (Lepidoptera: Noctuidae), In Relation To Rain And Wind" en Bulletin of Entomological Research, 73: 315-319.

Waters, D. A. 1996. The Peripheral Auditory Characteristics Of Noctuid Moths: Information Encoding And Endogenous Noise, en The Journal of Experimental Biology, 199: 857-868.

\section{Referencias de dispositivos electrónicos:}

http://www.murata.com/catalog/p15e6.pdf

http://www.sibalco.com/de/pdfs/obo/OboPro2_builtindrive_Buzzer.pdf

Ultrasonic instruments: Http://www.matec. com/instruments.htm 